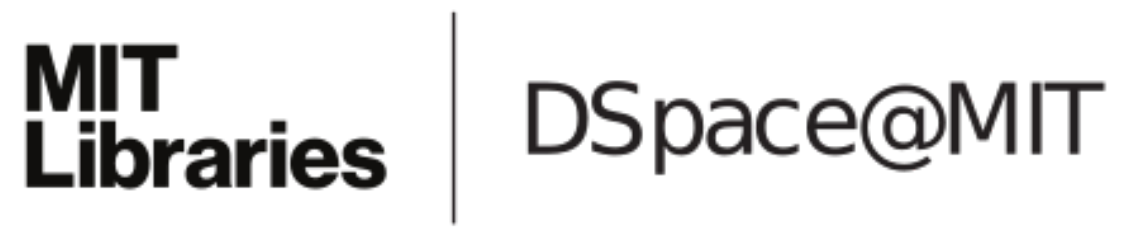

\author{
MIT Open Access Articles
}

Passive Internalization of Bioactive \#-Casein Peptides into Phospholipid (POPC) Bilayers. Free Energy Landscapes from Unbiased Equilibrium MD Simulations at $\mu$ s-Time Scale

The MIT Faculty has made this article openly available. Please share how this access benefits you. Your story matters.

As Published: https://doi.org/10.1007/s11483-020-09651-x

Publisher: Springer US

Persistent URL: https://hdl.handle.net/1721.1/131997

Version: Author's final manuscript: final author's manuscript post peer review, without publisher's formatting or copy editing

Terms of Use: Article is made available in accordance with the publisher's policy and may be subject to US copyright law. Please refer to the publisher's site for terms of use. 


\section{Passive internalization of bioactive $\beta$-casein peptides into phospholipid (POPC) bilayers. Free energy landscapes from unbiased equilibrium MD simulations at $\mu$ s-time scale.}

Cite this article as: Eduardo Jardón-Valadez, Charles H. Chen, Mariano García-Garibay, Judith Jiménez-Guzmán, Martin B. Ulmschneider, Passive internalization of bioactive $\beta$ casein peptides into phospholipid (POPC) bilayers. Free energy landscapes from unbiased equilibrium MD simulations at $\mu$ s-time scale., Food Biophysics, doi: 10.1007/s11483-020$\underline{09651-\mathrm{x}}$

This Author Accepted Manuscript is a PDF file of a an unedited peer-reviewed manuscript that has been accepted for publication but has not been copyedited or corrected. The official version of record that is published in the journal is kept up to date and so may therefore differ from this version.

Terms of use and reuse: academic research for non-commercial purposes, see here for full terms. http://www.springer.com/gb/open-access/authors-rights/aamterms-v1 
Passive internalization of bioactive $\beta$-casein peptides into phospholipid (POPC) bilayers. Free energy landscapes from unbiased equilibrium MD simulations at us-time scale.

Eduardo Jardón-Valadez ${ }^{1 *}$, Charles H. Chen ${ }^{2,3}$, Mariano García-Garibay ${ }^{4,5}$ Judith Jiménez-Guzmán ${ }^{4}$ Martin B. Ulmschneider ${ }^{2}$

'Departamento de Recursos de la Tierra. Universidad Autónoma Metropolitana, Unidad Lerma. Av. De las Garzas 10. Col. El Panteón. Lerma, Estado de México, México. C. P. 52005

${ }^{2}$ Department of Chemistry, King's College London, London SE1 1DB, United Kingdom.

${ }^{3}$ Synthetic Biology Group, Research Laboratory of Electronics, Massachusetts Institute of Technology, Cambridge, MA 02139, USA

${ }^{4}$ Departamento de Ciencias de la Alimentación. Universidad Autónoma Metropolitana, Unidad Lerma. Av. De las Garzas 10. Col. El Panteón. Lerma, Estado de México, México. C. P. 52005

${ }^{5}$ Departamento de Biotecnología. Universidad Autónoma Metropolitana, Unidad Iztapalapa. Av. San Rafael Atlixco 146, Col. Vicentina, Ciudad de México, México. C. P. 09340

*Corresponding author: h.jardon@correo.ler.uam.mx

Orcid: https://orcid.org/0000-0003-3701-429X

\section{Acknowledgements}

C.H.C. was supported by KCL PhD scholarships. Authors thank to Universidad Autónoma Metropolitana for providing computer time in supercomputing facilities: cluster Yoltla, and cluster Axolotl.

\section{Declarations}

\section{Funding}

No funding was received to assist with the preparation of this manuscript.

\section{Conflicts of interest/Competing interests}

The authors have no financial or proprietary interests in any material discussed in this article.

\section{Availability of data and material}

All relevant data is provided in the manuscript and the Online Resource.

\section{Code availability}

'Not applicable' 


\begin{abstract}
Absorption of bioactive peptides in the intestinal epithelium take place in the apical or the basolateral tight junctions of the cells. Depending on the peptide size and hydrophobicity, translocation mechanisms involve processes of passive diffusion, active transport by peptide-cotransporters such as members of the PepT family, and transcytosis by internalization vesicles. In this work, we investigated passive diffusion of bioactive peptides of 6,17 , and 30 amino acids into lipid bilayers of (POPC) phospholipid molecules. We initially selected these three peptides because such fragments are produced by partial hydrolysis of $\beta$ casein (BCN), and because of their physiological functions: BCN6 is an agonist of opioid receptors; $\mathrm{BCN} 17$ is an inhibitor of thrombin and angiotensinconverting enzymes, and BCN30 promotes secretion of the protective mucin barrier in the intestine. Our computational set up consisted of unbiased equilibrium molecular dynamics simulations, at the $\mu$ s-time scale, using an allatom force field. Each peptide was allowed to freely fold and unfold, as well as enter and exit the lipid bilayer, which allows determination of peptide affinity for the bilayer interface and hydrophobic core. Passive internalization of BCN6 (YPVEPF), BCN17 (YQEPVLGPVRGPFPIIV), and BCN30 (GVSKVKEAMAPKHKEMPFPK YPVEPFTESQ) displayed different dynamics at the bilayer interface: the BCN6 peptide attached and detached throughout the simulation trajectory; BCN17 and BCN30 attached irreversibly to the bilayer interface, respectively, with $\mathrm{N}$ - and $\mathrm{C}$-terminus fragments in close contact with lipid molecules. Quenching of tyrosine fluorescence data suggest interfacial interactions of BCN6, BCN17 and BCN30 in POPC lipid bilayers, consistent with the proposed modeling set up. This approach gave valuable information of peptide insertion and folding at a lipid bilayer, allowing to explore the initial stages of the peptide adsorption at the interface, and providing a model for evaluation of amphipathic properties of potential biofunctional peptides.
\end{abstract}

Keywords: neocasomorphin; $\beta$-casein hydrolysate; membrane active peptides;

POPC lipid bilayers; free energy landscape; tyrosine fluorescence probing.

\title{
Introduction
}

Milk proteins are a rich source of bioactive peptides that display antimicrobial, antithrombotic, antioxidant, immunomodulatory, opioid, and anticancer properties [1-4]. Bioactive peptides initially encrypted in the protein primary structure, can be exposed upon hydrolysis during digestion or fermentation processes, where protease or peptidase enzymes cleave the peptidic chain into 
smaller fragments from which active peptides are released. These fragments typically have lengths of 2 to 20 amino acids [1]. Absorption of bioactive peptides in the intestinal epithelial cells (IEC), which cover the inner side of the intestine tract, take place at the apical or the basolateral sides according to the following proposed mechanisms [5]. The microvilli of the apical membrane, also called brush-border membrane, contain transporters such as members of the PepT family [6] that specifically translocate di- and tripeptides, from the small intestine to the blood circulation [5]. Satake et al. [7] found that the tripeptide Val-Pro-Pro was assimilated intact by human intestinal Caco-2 monolayer cells, which suggested a paracellular transport mechanism. Paracellular diffusion is a passive transport pathway mediated through the intercellular junctions between IEC, important for mineral permeation [5]. Transcytosis was also proposed for intact protein assimilation by means of endocytic vesicles, which is mainly a degradative pathway by lysosomal processing; alternatively, only $10 \%$ of intact proteins get across by a non-degradative pathway of transcytosis [8]. Finally, passive permeation of hydrophobic molecules through the membrane of IEC was described [9].

In this work, we investigated the passive diffusion of bioactive peptides of 6,17 , and 30 amino acids produced through partial hydrolysis of $\beta$-casein (BCN). Our objective was to calculate, from all-atom molecular dynamics simulations, free energy profiles of peptides that passively diffused to a membrane bilayer of 1-palmitoyl-2-oleoyl-sn-glycero-3-phosphocholine (POPC) lipid molecules. Our set up was designed to explore the peptide conformational changes and lipid interactions that could favor the internalization of the peptide into the lipid bilayer. 
$\beta$-casomorphins (BCM) are agonist of $\mu$-opioid receptors, located in the central and peripheral nervous system, as well as in endocrine and immune systems [10]. Nomenclature of this group of peptides refers to $\beta$-casein ( $\beta$ caso), the precursor protein, and morphine refers to the Greek god of sleep, Morphus [4].

From the hydrolysis of $\mathrm{A} 1 \mathrm{BCN}$ variant, with a histidine at position 67 in type A1 milk, BCM are produced by enzyme hydrolysis [4]. Regarding the physiology of the BCM peptides results are rather controversial. On the one hand, in diabetic rats, BCM-7 was associated with an increase of plasma insulin, a decrease of glucagon, and enhanced antioxidant protection of kidney tissue [11]. On the other, epidemiological data analysis showed significant correlations between consumption of type $\mathrm{A} 1 \mathrm{BCN}$ and ischemic heart disease [12]. Jinsmaa et al. [13] reported the $\beta$-neocasomorphin as a bioactive peptide produced by in vitro hydrolysis with trypsin or pepsin, and found that this fragment was an agonist of the $\mu$-opioid receptor.

Interestingly, the $\beta$-neocasomophin with sequence YPVEPF (BCN6) was related to a exorphin $\mathrm{C}$ of gluten YPISL, also an opioid agonist isolated from digestion of wheat gluten, both having an aliphatic side chain amino acid at the third position, with a potent activation response of the $\mu$-opioid receptor [13]. In our study, we analyzed the interaction of the BCN6 peptide in a POPC lipid bilayer to determine how these peptides absorb and get taken up in the digestive tract. 
The BCN fragment 193-209, sequence YQEPVLGPVRGPFPIIV, of 17 amino-acids (BCN17) has shown antithrombotic, antihypertensive, [14] and immunomodulatory properties [15]. Even though peptidases are present all along the small intestine, with increasing longitudinal activity from pylorus to ileum [16], when peptides with more that 20 amino acids endure hydrolysis through the small intestine, degradation efficiency of large peptides is rather slow in comparison to degradation of smaller peptides [17]. Studies on BCN17 transport in a Caco-2 cells monolayer, a typical model for studying transport in human enterocyte cells, Regazzo et al. [15] proposed a transcytosis mechanism as peptidase enzymes of the apical villi were not able to hydrolyze the BCN17 peptide, beyond the first and last amino acids, producing BCN (193-208) and BCN (194-209). In this work, we generated a molecular dynamics (MD) trajectory for the BCN17 peptide to evaluate its lipid bilayer interactions, as it was expected to diffuse to the membrane environment due to its amphipathic properties provided by aromatic, aliphatic and charged side chain residues.

The BCN fragment 94-123, sequence GVSKVKEAMAPKHKEMPFPKYP VEPFTESQ, with 30 amino acids (BCN30), increases expression of protective mucin proteins, MUC2 and MUC4, in Goblet cells [18]. Intestinal mucus gel contains high-molecular-weight glycoproteins and forms a barrier that limit the access of pathogens. Therefore, the BCN30 showed multifunctional properties as supplement in commercial applications of yoghurts [19]. In this work, we study the interaction of BCN30 with lipid bilayers, in order to analyze how the peptide folds and internalizes into the hydrophobic membrane core. 
As an initial approximation to describe the $\mathrm{BCN}$ biofunctional peptides at membrane interfaces, we set up atomic detail molecular dynamics (MD) simulations using classical force fields. MD approaches have provided detailed descriptions of peptide insertion and peptide folding [20,21], as lipid bilayers provide a molecular environment that induces secondary structure in membrane-active peptides [22]. All-atom simulation methods include tools for studying structure and interactions of peptides at bilayer interfaces, in particular, when researchers are interested in disclosing molecular mechanisms involved in the peptide assimilation at model membranes. In experiments, on the other hand, the affinity of peptides to lipid bilayers has been measured for designed pentapeptides of type AcWL-X-LL-OH (for $X$ being any of the essential amino acids) in equilibrium with large unilamellar vesicles (LUV) of 1-palmitoyl-2oleoyl-sn-glycero-3-phosphocholine (POPC) lipids [23]. Experimental measurements led to a hydrophobicity scale in terms of the free energy for transferring an unfolded peptide from the aqueous solution to the lipid interface [23]. Because the extensive work done on bilayers of POPC, this system became a standard in biophysical experiments. Interestingly, reports of in vitro digestion of $\mathrm{BCN}$ showed that addition of phosphocholine vesicles into the simulated gastroduodenal digestion has an inhibitory effect on the proteolysis, in a concentration-dependent fashion [24, 25]. Therefore, studying the interactions of BCN peptides and phosphocholine lipid bilayers could provide some clues for understanding interactions between milk-derived membraneactive peptides and zwitterionic phospholipids as a simplified model of the gastrointestinal assimilation process for passive absorption of hydrophobic biofunctional peptides. 


\section{Methods}

System preparation. Three simulation boxes were prepared by the following procedure. The peptide initial coordinates were generated directly from the amino acid sequence using the PyMol software [26]. All peptides were defined with acetylated $\mathrm{N}$-terminus and methyl-amide $\mathrm{C}$-terminus. The BCN6 and BCN17 were defined in extended random coil conformation, and BCN30 was defined in $\alpha$-helix conformation; each peptide located initially in the solvent phase approximately $20 \AA$ away from the bilayer interface. Protonation of side chains was assigned according to the predominant state at $\mathrm{pH} 7$, namely, protonated arginine and lysine, unprotonated glutamate, and neutral histidine (N81-protonated, $\pi$ tautomer) [27]. An additional simulation box with protonated $\mathrm{N}$-terminus and unprotonated $\mathrm{C}$-terminus was prepared for the BCN6 peptide, for a contrast of the peptide dynamics against the neutral capped termini set up. The initial configuration for the POPC bilayers was generated through the membrane builder plugin of the VMD software [28], with the bilayer normal oriented along the $z$-axis, and including, respectively, 50, 72, and 98 lipid molecules for the BCN6, BCN17, and BCN30 simulation boxes. Water molecules were added, approximately 40 per POPC, using the solvate program of the GROMACS 2019 [29] package suite. Chloride and sodium ions were included for charge neutrality and molar concentration of $20 \mathrm{mM}$. The box height was set to $8 \mathrm{~nm}$ in all systems, while box dimensions in $x y$ plane were $3.97 \mathrm{~nm}$ containing 12971 atoms, $4.87 \mathrm{~nm}$ containing 19369 atoms, and 5.49 nm containing 24690 atoms, respectively, for the BCN6, BCN17, and BCN30 
systems. All initial configurations were energy minimized to avoid repulsive contacts due to random assignment of coordinates in the simulation box set up.

Molecular dynamics simulations. All simulation trajectories were generated using the GROMACS 2019 package suite. A preliminary equilibration in the NPT ensemble was carried out at $300 \mathrm{~K}$ and 1 bar using a weak temperature coupling [30] with a time constant of $0.1 \mathrm{ps}$, and a semi-isotropic weak pressure coupling [31] with a time constant of $0.1 \mathrm{ps}$ and compressibility of 4.6 $\times 10^{-5}$ bar $^{-1}$. Bonds involving hydrogen atoms were constrained using the LINCS algorithm [32]. Electrostatic interactions were calculated by the particlemesh Ewald (PME) method, with a $4^{\text {th }}$ order interpolation scheme. For nonbonding interactions, we used a $10 \AA$ cut-off, with Verlet scheme for neighbor searching, which takes advantage of GPU acceleration and tune load balance of the CPU/GPU by scaling the electrostatic cutoff and the grid spacing. At the preliminary equilibration stage, $50 \mathrm{~K}$ steps were performed using a time step of $1 \mathrm{fs}$ and updating the neighbor list every 5 steps. Next, an equilibration stage of $5000 \mathrm{~K}$ steps was performed, with 2 fs time step, at $313 \mathrm{~K}$ and $1 \mathrm{bar}$, and 20 steps for updating the neighbor list. The production run started from the last configuration of the previews equilibration stage, at $333 \mathrm{~K}$ and 1 bar at $5-8 \mu \mathrm{s}$ time scale, using the Parrinello-Rahman barostat [33] in semi-isotropic conditions, and the V-scale algorithm [30] for temperature control. Unbiased equilibrium $\mu$ s-timescale MD simulation trajectories were generated at $333 \mathrm{~K}$ and 1 bar. The advantage of this technique is that it allows capturing the equilibrium interactions of a single peptide with a lipid bilayer without imposing 
any additional force on top of the molecular interactions defined by the force field [34].

Hight temperature unbiased equilibrium peptide partitioning. For implantation of equilibrium MD peptide partitioning studies in water-lipid bilayer interfaces, at high temperature, Ulmschneider et al. [34] proposed as necessary conditions: I. Increase of temperature has no effect on the peptide insertion propensity into the lipid bilayer; II. The peptide must be thermostable, so the native fold is preserved at high temperature; III. The liquid crystal phase $(L \alpha)$ of the membrane must be preserved at high temperature, and the chemical transbilayer composition remains fundamentally unchanged even as the bilayer is heated and reduces in density. In such conditions, thermodynamics and kinetics of peptide insertion into bilayers rely on convergence of simulation trajectories. In hydrophobic peptides, all-atom simulation demonstrated that the peptide $A c e-A_{4}(L A)_{5} A_{4}-A m e$, initially unfolded, folds and inserts into the bilayer core within 300 ns of trajectory at $80^{\circ} \mathrm{C}$ [34]. In addition, in W16 and W23 peptides, enthalpy for the insertion in a two-state process, surface-bound (SB) to transmembrane (TM), were comparable to experimental measurements at room temperatures. Therefore, in membrane spanning hydrophobic peptides, simulations at high temperature preserved thermodynamic and kinetic features of folding and internalization, with advantage of reducing transition time by orders of magnitude (50-500 times). The experimental thermostability of these and other membrane active peptides was confirmed experimentally using circular dichroism spectroscopy melting scans. Key to this thermostability is the $\sim 5 \mathrm{kcal} / \mathrm{mol}$ penalty of breaking a helical hydrogen bond in the hydrophobic 


\section{AUTHOR ACCEPTED MANUSCRIPT}

membrane core. Even at the membrane interface this penalty is still $\sim 2 \mathrm{kcal} / \mathrm{mol}$ per bond [35]. Previews implementations included melittin (GIGAVLKVLTTGLPALISWIKRKRQQ), which showed irreversible adsorption into the interface of POPC bilayers [36]. Even though it was not possible to calculate the binding free energy due absence of unbound states, thermostability of the helical folding was evaluated by simulation and CD titration experiments, using POPC LUV; results suggested that melittin was stable at high temperature $\left(90^{\circ} \mathrm{C}\right)$, strongly bound to the bilayer interface at the glycerol-carbonyl linker height, and oriented perpendicular to the bilayer normal [36]. Lipid bilayers interfaces provide an amphiphilic environment that promotes secondary structure in peptides [22], and MD simulations provide methods to detect peptide affinity to bilayers, and the interactions leading to folding, adsorption or insertion mechanisms. In our implementation, since the native fold of the BCN peptides were not known, we assumed feasible to generate converged simulation trajectories at microsecond time scale for the BCN6 peptide; for BCN17 and BCN30 peptides, we generated $\mu$ s time scale trajectories to determine the population of bound/unbound states, and to describe the peptide conformational dynamics at the bilayer interface.

Potential energy functions. The CHARMM36 parameter set for the peptide atoms were used [37-39], with CMAP correction for the peptide atoms [40]. The POPC lipids parameters were taken from reference [41], and the TIP3P model was used for the water molecules [42]. 
Trajectory analysis. The conformational landscapes of BCN6, BCN17, and BCN30 were obtained by principal component analysis of the dihedral angles, using a linearized transformation of the $\phi$ and $\psi$ angles [43], and the covariance matrix implementation of the GROMACS 2019.4 suite of programs [29, 44]. By diagonalization of covariance matrices, the resultant eigenvectors define a transformed coordinate system for the principal modes of motion, and the eigenvalues corresponding to the mean square fluctuation in direction of the principal modes. As a measure of the total fluctuation, the root mean square fluctuation (RMSF) was calculated from the square root of the sum of eigenvalues divided by the number of atoms. Cosine content was calculated for the first four principal components (PC) as a measure of the contribution of random thermal diffusion, with values in the interval 0 to 1 , respectively, for nocosine content or full cosine content due to random thermal fluctuations [45]. Trajectories were divided blocks of $2^{5}-2^{15}$ time frames (collected every $200 \mathrm{ps}$ ) for evaluation of the convergence of total fluctuation of dihedral PCA eigenvalues. Mass distributions for lipid phosphates and carbonyl groups, as well as terminal methyls of the acyl chains were calculated as function of the bilayer normal, for identification of the bilayer interface and hydrophobic core regions. In addition, order parameters $\mathrm{S}_{\mathrm{CH}}$ for methylene groups, defined as $\mathrm{S}_{\mathrm{CH}}=\left\langle 3 / 2 \cos ^{2} \theta-1 / 2\right\rangle$, were calculated and compared against ss-NMR data for labeled acyl chains in POPC multilamellar vesicles [46]. Visualization of the trajectories was performed using the VMD software [28], as well as in house analysis scripts developed for specific calculations, such as peptide-lipids contacts, center of mass distance to the bilayer core, among other tools. 
Tyrosine fluorescence probe. $\mathrm{BCN6}, \mathrm{BCN} 17$, and $\mathrm{BCN} 30$ peptides were synthesized using solid-phase peptide synthesis and purified to $98 \%$ using reversed phase HPLC with C18 column. Peptide purity and identity were confirmed by HPLC and ESI mass spectrometry. 1-palimitoyl-2-oleoyl-snglycero-3-phosphocholine (POPC) was purchased from Avanti Polar Lipids. Lipid was dissolved in chloroform, mixed, and dried under nitrogen gas in a glass vial. Remaining chloroform was removed under vacuum overnight. Lipids were resuspended in $10 \mathrm{mM}$ sodium phosphate buffer ( $\mathrm{pH}$ 7.0). LUV were made by extruding the lipid suspension 10 times through $0.1 \mu \mathrm{m}$ nucleopore polycarbonate filters to give LUV of $100 \mathrm{~nm}$ diameter [47]. $50 \mu \mathrm{M}$ peptides and varied concentrations of POPC LUV (lipid concentration: 0, 10, 20, 40, 78, 156, $313,625,1250,2500,5000$, and $10000 \mu \mathrm{M})$ were prepared in $10 \mathrm{mM}$ phosphate buffer $(\mathrm{pH} 7.0)$. The mixtures were measured after $1 \mathrm{hr}$. Excitation was fixed at $274 \mathrm{~nm}$ (slit $9 \mathrm{~nm}$ ) and emission was collected from 300 to $400 \mathrm{~nm}$ (slit $9 \mathrm{~nm}$ ). The spectra were recorded using Cytation ${ }^{\mathrm{TM}} 5$ Cell Imaging MultiMode Reader from BioTek and were averaged by 3 scans.

\section{Results}

Three peptides encrypted in the BCN primary structure were chosen for this study: BCN6, an agonist of opioid receptors [13]; BCN17 an inhibitor of thrombin and angiotensin-converting enzyme [14]; and BCN30 that promotes secretion of mucin barrier in the intestine [19]. To evaluate membrane activity each peptide was set up in a simulation box containing a POPC lipid bilayer, and solvent molecules for hydration of the lipid heads ( 40 waters per lipid). 
Unbiased $\mu$ s-timescale equilibrium MD simulation trajectories were generated at $333 \mathrm{~K}$ and $1 \mathrm{bar}$. The advantage of this technique is that it allows capturing the equilibrium interactions of a single peptide with a lipid bilayer without imposing any additional force on top of the molecular interactions defined by the force field [34]. Using high temperature, peptide-membrane biding free energy is estimated under equilibrium conditions, if both bound and unbound states are populated; conversely, if the peptide is irreversible bound, no free energy can be calculated.

Our simulation protocol allowed us to identify the population of bound and unbound states of the BCN peptides at the bilayer interface. All peptides were initially placed $>20 \AA$ away from the bilayer surface, in the aqueous solution. The initial peptide conformation for the BCN6 and BCN17 systems was random coil, whereas a helical conformation was defined for the BCN30 peptide to fit it into the simulation box. All simulation trajectories were generated allowing the peptide to freely fold and unfold, as well as enter and exit the bilayer (Figure 1). In previews high temperature simulations, on melittin and POPC bilayers at $80^{\circ} \mathrm{C}$, the peptide bind to the bilayer interface and folded as a stable $\alpha$-helix [36]. Lipid bilayers provide a molecular environment that promote formation of secondary structure in peptides [22], hence we could also identify signatures of secondary structure formation in the BCN17 and BCN30 peptides (Online Resource). In principle, as our simulations covered several microseconds, the final state is independent of the initial peptide structure; nonetheless, BCN peptides must be further examined in both experimental and simulation studies. 
Affinity of the BCN peptides to bilayer interface. We have previously shown that polyleucine derived transmembrane segments and membrane active peptides [20], such as melittin from honey bee venom [36, 47], and maculatin [48], adsorb rapidly and irreversibly to lipid bilayers. To evaluate whether partial hydrolysis of BCN produces peptides with potential membrane activity, we calculate the relative distance between the bilayer hydrophobic core, centered at $\mathrm{Z}=0 \AA$, and the center of mass (COM) of BCN6 peptide, and $\mathrm{C}$ - and $\mathrm{N}$ termini of BCN17 and BCN30 (Figure 2). Data for the protonated N- and unprotonated C-terminus BCN6 peptide (Online Resource) showed similar behavior in bound and unbound transitions, as in Figure $2 \mathrm{~A}$, with a detectable difference in the population of bound and unbound states, which can be explained by the increased electrostatics of the charged termini BCN6.

From a preliminary comparison of the internalization dynamics, we could highlight some differences in the behavior of the BCN peptides at the bilayer interface (Figure 2). For example, BCN6 was highly dynamic, entering into the hydrophobic core and emerging up to the solvent bulk (Figure 1A, 2A). Bilayer bound-unbound transitions of BCN6 throughout the MD trajectory allowed us to calculate the free energy profile for the peptide's hydrophobic affinity (see Peptide Hydrophobicity Profile). Internalization of BCN17 was driven by the of C-terminus, as this side inserted into the bilayer (10-15 $\AA$ ) within the first 500 ns of trajectory; the N-terminus, on the other hand, located in the bulk solvent, and experienced bound-unbound transitions in the 500 ns time scale (Figure 1-2B). Internalization of $\mathrm{BCN} 30$ was driven by the $\mathrm{N}$-terminus, which during the first 3 us of trajectory was located at $10 \AA$ from the bilayer center; subsequently, the $\mathrm{N}$-terminus displaced to the interface at $20 \AA$, and finally reinserted to the 
bilayer for the last $500 \mathrm{~ns}$. The C-terminus of $\mathrm{BCN} 30$ mostly remained in the bulk solvent, although this terminus also explored the lipid interface at $15 \AA$ from the bilayer center (Figure 1-2C). In summary, the short peptide BCN6 diffused freely from the aqueous region to the bilayer and vice versa, while BCN17 and BCN30 both bound irreversibly to the bilayer, although with opposite bilayer affinities regarding the $\mathrm{N}$ - and $\mathrm{C}$-terminus of these larger $\mathrm{BCN}$ peptides.

Bilayer mass distributions and lipid order parameters. The lipid bilayer of POPC molecules provides an amphipathic environment with the lipid heads facing the aqueous solution and the lipid tails forming the hydrophobic core. The bilayer was defined in the $x-y$ plane, and the bilayer normal corresponds to the $z$ coordinate. Figure 3 shows the mass density of phosphates and carbonyls, respectively, located at the head groups and the acyl linker; mass density distribution of terminal methyl groups has a maximum at $z=0 \AA$, which is the center of the bilayer, right in the middle of the hydrophobic core. Along the bilayer normal, the transition from the hydrophobic core to the aqueous phase starts at $\sim 10 \AA$, with the interface region within 14-25 $\AA$, including the carbonyl and phosphate distributions. At $z=30 \AA$, water reached the bulk solvent density (9.6 $\mathrm{g} \mathrm{cm}^{-3}$, Online Resource). As mentioned before, a necessary condition to preserve thermodynamics and kinetics in peptide partitioning simulations is that the chemical composition across de bilayer must be preserved at high temperatures. Since all simulations were performed at 333K, in Figure 3 we confirmed that all distributions agreed with previous reports for POPC bilayers [20].

Interactions of $\mathrm{BCN}$ peptides and the membrane were mostly observed at the bilayer interface level. BCN17 and BCN30 bound irreversibly to the 
interface, respectively, with the $\mathrm{C}$-terminus and the $\mathrm{N}$-terminus making contacts with lipid heads (Figure 2). Order parameters $S_{C H}$ were calculated for those lipids in close contact with the BCN17 and BCN30. For the BCN6, calculation of $\left|\mathrm{S}_{\mathrm{CH}}\right|$ was done over all the POPC lipids, as no permanent contacts were observed. Figure 4 shows the order parameter $\left|\mathrm{S}_{\mathrm{CH}}\right|$ along the acyl chains, from C2-C16 of sn1 and sn2 tails. For comparison, the ss-NMR order parameters were included [46]. The calculated profiles of the $\left|\mathrm{S}_{\mathrm{CH}}\right|$ along with the sn1 and sn2 tails, were in good agreement with experimental measurements, which confirms that the bilayer structure was well preserved at $333 \mathrm{~K}$. Due to the lack of structural information of the BCN peptides here studied, the peptide structural stability at high temperature was not tested. Nonetheless, our computational approach allowed us to determine how the BCN peptides behave at membrane interfaces with amphipathic properties well preserved at $333 \mathrm{~K}$, for an initial elucidation of mechanisms involved in the peptide adsorption at lipid interfaces.

Peptide Hydrophobicity Profile. In a previous section, we identified bound and unbound states of the BCN peptides using a distance criterion. For the BCN6 peptide two states were populated, as this peptide showed permanent bound and unbound transitions. In peptides $\mathrm{BCN} 17$ and $\mathrm{BCN} 30$, transitions were calculated for fragments, namely, N-terminus, C-terminus, and a middle fragment defined only for BCN30 (Table 1). For each peptide fragment, histograms for distance COM to bilayer center were calculated, for bins $1 \AA$ width. Free energy profiles were calculated from histograms according to $\Delta \mathrm{G}=-$ $k_{B} T \operatorname{In}\left[P / P_{\max }\right][20,43]$, with the $P_{\max }$ corresponding to the maximum probability state (Figure 5). The minimum of free energy for the BCN6 peptide was found at 
$17 \AA$, and the second minima of $1.78 \mathrm{~kJ} / \mathrm{mol}$ at $32 \AA$, both minima connected by a $3.8 \mathrm{~kJ} / \mathrm{mol}$ barrier at $27 \AA$. The presence of two minima was expected from the bound and unbound transitions throughout the trajectory, consistent with a relatively low free energy barrier (Figure 5A).

Free energy profiles for the $\mathrm{C}$-terminus of $\mathrm{BCN} 17$ revealed a minimum at $14 \AA$, while the $\mathrm{N}$-terminus minimum was found at $22 \AA$ (Figure 5B). Such difference in the bilayer affinity of the C-terminus and N-terminus of BCN17 resembled a peptide with amphipathic properties [49], which can be explained by the presence of polar side chain groups in the first half (YQEPVLGPV) versus the predominantly non-polar side chain groups in the second half (RGPFPIIV).

In BCN30 all fragments were mainly attached to the bilayer interface, with free energy minima at 18,21 , and $24 \AA$, respectively, for the $\mathrm{N}$-terminus, middle fragment, and C-terminus. BCN30 fragments showed broad probability distributions with only one prominent maximum in the COM-bilayer distance histograms, consistent with an irreversible bilayer interface binding (Figure 5C). Interestingly, BCN30 showed partial $\alpha$-helix folding at the $\mathrm{N}$-terminus fragments, including amino acids VSKVKEA, while the middle fragment and C-terminus showed transitions between turns and bends (Online Resource).

From a comparison of the free energy profiles of all BCN fragments, we found differences regarding peptide and bilayer interactions: i) in contrast to the $\mathrm{BCN6}$, the largest peptide $\mathrm{BCN} 30$ was attached permanently to the bilayer interface with only one prominent free energy minimum, provided that no bilayer bound and unbound transitions were observed however the amino acid 
sequence of the BCN30 fragments; ii) In contrast to the amphipathic behavior of $\mathrm{BCN} 17$, the peptide affinity of BCN30 was similar for all fragments, therefore, BCN30 was not identified as a peptide with amphipathic properties. The behavior of the $\mathrm{BCN} 30$ at the bilayer interface, nonetheless, may be a consequence of its folding dynamics, which can be evaluated from internal coordinates such as dihedral angles of the backbone atoms, as described in the next section. Since essentially only bound states were found in the BCN17 and $\mathrm{BCN} 30$, suggesting a very strong interaction to the lipid interface for these larger peptides, the convergence of trajectories was not reached at the multimicrosecond time scale, as we describe in the calculation of total fluctuation in the dihedral PCA analysis (Online Resource).

Conformational Dynamics of the BCN Peptides. The behavior of the BCN peptides at the bilayer interface was well distinguished by different characteristics: i) fast bound and unbound transitions as in BCN6; ii) amphipathic properties as observed in BCN17; iii) stable motifs of secondary structure as in BCN30. From the amino acid sequence, it was expected that membrane binding was driven by aromatic or non-polar side chains. Nonetheless, peptide folding could play a role in the peptide internalization, as observed for other membrane active peptides [21, 48]. Therefore, we analyzed the peptide conformations that could promote the internalization of the $\mathrm{BCN}$ peptides into lipid bilayers. For this purpose, we analyzed dihedral principal components (dPCA) using tools implemented in GROMACS 2019 [44]. Principal components of the backbone dihedral angles indeed capture the roughness of the conformational landscape of small peptides [43]. Following the backbone 
chain from the $\mathrm{N}$ - to the $\mathrm{C}$-terminus, the angle of $\phi_{\mathrm{i}}$ corresponds to the dihedral of $C_{1-i}, N_{i}, C A_{i}, C_{i}$ atoms, and the angle $\psi_{i}$ corresponds to the dihedral of $N_{i}, C A_{i}$, $\mathrm{C}_{\mathrm{i}}$, and $\mathrm{N}_{\mathrm{i}+1}$ atoms [50]. Figure 6 shows distributions for dihedral $\left(\phi_{i}, \psi_{\mathrm{i}}\right)$ angles for the BCN peptides. Altis et al. [51] proposed a transformation from $\left(\phi_{i}, \psi_{i}\right)$ to $\left\{x_{n}=\cos \left(\phi_{n}\right), y=\sin \left(\phi_{n}\right)\right\}$, which account for the circular statistics of angular variables by defining differences with the usual Euclidean metric: $d^{2}=\Delta x^{2}+\Delta y^{2}$. Therefore, covariance matrices were calculated for the transformed coordinates $\left(\mathrm{x}_{\mathrm{n}}, \mathrm{y}_{\mathrm{n}}\right)$, for $\mathrm{n}$ dihedral angles. Figure 7 shows distributions dihedral principal components (dPC1-6) for the of BCN6 peptide. Multimodal distributions were detected in dPCA1-4, therefore, projections of the free energy landscape revealed at least 5 energy minima in the $\mathrm{dPC} 1$ and defined conformational clusters in bi-dimensional projection plots of dPC1-dPC2 and dPC3-dPC4 (Figure 8). Importantly, the total fluctuation was calculated for the BCN6, and fragments of $\mathrm{BCN} 17$ and $\mathrm{BCN} 30$, as the square root of the sum of the eigenvalues divided by the number of atoms, which for dihedral PCA analysis, corresponds to $2 n / 3$ for $n$ angles in the set $\left\{\phi_{1}, \phi_{2}, \ldots \psi_{n-1} \psi_{n}\right\}$. Figure $S 4$ (Online Resource) shows that the total fluctuation, calculated as function of block size, for each trajectory divided in $2^{5}-2^{15}$ time frames; only the BCN6 reached convergence. In contrast, none of the fragments of BCN17 or BCN30 display a similar behavior in the total fluctuation. A converged total fluctuation, as that found in BCN6, suggests that for a given block size, fluctuations of the transformed $\mathrm{x}$ and $\mathrm{y}$ coordinates include all the variability of such coordinates. Conversely, when the total fluctuation increases along with the block size, then the total fluctuation requires longer trajectories to converge. From this result, only for the BCN6 the projection of the of the dPC1 and the distance BNC6- 
bilayer was calculated to classify those conformations that favor peptide internalization into the lipid bilayer (Online Resource).

Due to the larger size of the BCN17 and BCN30 peptides in comparison to BCN6, we followed the folding and unfolding dynamics at the bilayer interface by calculation of the $\mathrm{H}$-bond patterns, as defined in the dictionary of protein secondary structure [52]. Significant secondary structure motifs were detected, with rapid transitions between turn and bend throughout the whole backbone of BCN17 (Online Resource). Similar turn and bend transitions were observed in BCN30 in the segment A103 to Q123, whereas V95 to A101 formed a persistent $\alpha$-helical fold at the $\mathrm{N}$-terminal side (Online Resource).

Peptide adsorption at the lipid interface by tyrosine fluorescence probe. All BCN6 (sequence: YPVEPF), BCN17 (sequence: YQQEPVLGPVR GPFPIIV), and BCN30 (sequence: GVSKVKEAMA PKHKEMPFPK YPVEPFTESQ) have one tyrosine in their peptide sequences. It can be utilized as a fluorescent probe [53] to study its interaction with the target membrane, i.e., POPC LUV. We evaluated these three peptides (50 $\mu \mathrm{M}$ peptide concentration) against a varied range of POPC LUV from 0 to $10000 \mu \mathrm{M}$ and recorded their spectra using the fluorometer. The results showed that the maximum wavelength and maximum fluorescence intensity were all similar between different lipid concentrations (Figure 9), which did not have a blue shift or significant change of the maximum fluorescence intensity. It suggests that the tyrosines in these peptides did not penetrate into or stayed in the hydrophobic core of the membrane. This finding agreed with the MD simulations (Figure 1-2) because the tyrosines were either barely staying at the membrane interface or in the aqueous solution. Remarkably, the experimentally validated MD simulations provide atomic details 
on dynamics and how these peptides interact with the POPC bilayer whenever the absorption phenomenon is difficult to observe in experiments.

\section{Discussion}

The behavior of three BCN peptides with 6,17 , and 30 amino acids, at bilayer interfaces of POPC phospholipids, was investigated by a computational set up using an all-atom force field and unbiased MD simulation in the isothermalisobaric ensemble, at $333 \mathrm{~K}$ and 1 bar. In addition, to verify the peptide and bilayer interactions, we took advantage of quenching of tyrosine fluorescence in folded domains, and tyrosine fluorescence enhancement in unfolded segments [54]. Experimental measurements and the MD simulation results suggested interfacial bound states, i.e., BCN peptides inserted up to the bilayer interface, showing different dynamics regarding bound-unbound transitions, backbone dihedrals, and side chain contacts to lipid heads. Even though we found specific peptide interactions and dynamics at the bilayer interface, our computational benchmarks suggest that for the BCN17 and BCN30 peptides, simulations would require trajectories $10-100$ times longer for a proper description of the peptide's dynamics, which are currently beyond the computational resources available.

Studies on folding and partitioning of membrane active peptides include MD methods, which provide valuable tools for addressing aspects of the peptide assimilation into bilayers. Processes such as $\alpha$-helix folding at the interface [36], $\alpha$-helix insertion into a lipid bilayer [21], assembly of antimicrobial maculatin in the hydrophobic core [48], insertion free energy calculations [55], among others, show the broad applications of MD methods, at increased 
temperature, for a detailed description of membrane and peptide interactions. By setting an increased temperature MD protocol, in this work, we analyzed simulation trajectories to explore adsorption and assimilation processes of biofunctional peptides derived from partial hydrolysis of type A1 BCN. Our main assumption was that passive diffusion and interfacial insertion into a bilayer membrane, may serve as a discriminant behavior to identify relevant peptide properties upon absorption in epithelial intestinal cells [5, 56]. Even though membrane translocation was not observed at the multimicroseconds timescale, we could identify some differences in the behavior of BCN peptides and recognize the relevance of the physicochemical properties of the amino acids. For example, the symmetry of the BCN6 composition YPEVPF, with two aromatic side chain groups, enhanced the membrane partitioning dynamics as this peptide could enter the bilayer with both the $\mathrm{N}$-terminus or the $\mathrm{C}$-terminus, with a low free energy barrier given the similar hydrophobicity of tyrosine and phenylalanine [23]. In contrast, BCN17 insertion was driven by the hydrophobic C-terminus, while the $\mathrm{N}$-terminus remained chiefly located in the aqueous region with only transient membrane internalization. Because of the different bilayer affinity of the $\mathrm{N}$-and $\mathrm{C}$-terminus, BCN17 was identified as amphipathic peptide, which could be a functionality of interest in specific applications [49]. The largest peptide studied here, BCN30, showed irreversible adsorption with a stable $\alpha$-helical secondary structure at the $\mathrm{N}$-terminus side, including VSKVKEA amino acids, and located in the interface at $18 \AA$ from the bilayer center. The tyrosine fluorescence measurements confirmed that both BCN17 and BCN30 bound to the membrane interface, in unstructured conformations. In our multimicrosecond time scale simulations, we detected unstructured 


\section{AUTHOR ACCEPTED MANUSCRIPT}

conformations in the BCN peptides at the interface of POPC bilayers, except for helical domain at the $\mathrm{N}$-terminus of $\mathrm{BCN} 30$. As a possible explanation, we noticed that the tyrosine in $\mathrm{BCN} 30$ belongs to the $\mathrm{C}$-terminus fragment, which located in average at $24 \AA$ from the bilayer center, whereas the $\mathrm{N}$-terminus located at $18 \AA$; thus, the tyrosine was closer to the aqueous phase, which is consistent with the tyrosine fluorescence probing results for the BCN30 peptide.

\section{Conclusions}

Our computational approach was designed to evaluate the behavior of peptides at phospholipid membrane interfaces, to describe the mechanism of hydrophobic assimilation of products of the partial hydrolysis of $\beta$-casein. A similar approach can be implemented for hydrolysates from other sources as whey proteins, or gluten. In particular, for identification of peptides that could be applied as additives in the food industry, taking advantage of the amphipathic properties as well as the biological functionality by triggering cellular responses in the intestinal tract. 
Table 1. BCN peptide amino acid sequences and fragments defined for the calculation of the bound and unbound states.

\begin{tabular}{ccccc}
\hline Name & $\begin{array}{c}\text { Peptide } \\
\text { Sequence }\end{array}$ & N-terminus & M-term & C-terminus \\
\hline BCN6 & YPVEPF & & & \\
BCN17 & $\begin{array}{c}\text { YQEPVLGPVR } \\
\text { GPFPIIV }\end{array}$ & YQEPVLGPV & & RGPFPIIV \\
BCN30 & GVSKVKEAMA & GVSKVKEAMA & PKHKEMPFPK & YPVEPFTESQ \\
& PKHKEMPFPKY \\
& PVEPFTESQ & & & \\
\hline
\end{tabular}

\section{References}

1. D. P. Mohanty, S. Mohapatra, S. Misra and P. S. Sahu, Saudi J Biol Sci 23 (5), 577-583 (2016).

2. B. N. P. Sah, T. Vasiljevic, S. McKechnie and O. N. Donkor, COMPR REV FOOD SCI F 14 (2), 123-138 (2015).

3. H. S. Gill, F. Doull, K. J. Rutherfurd and M. L. Cross, Br. J. Nutr. 84 (S1), 111117 (2000).

4. M. R. u. Haq, R. Kapila, U. K. Shandilya and S. Kapila, Int. J. Food Prop. 17 (8), 1726-1741 (2014).

5. M. Shimizu, Biosci. Biotechnol. Biochem., 232-241 (2010).

6. F. Guettou, E. M. Quistgaard, M. Raba, P. Moberg, C. Löw and P. Nordlund, Nat. Struct. Mol. Biol. 21 (8), 728-731 (2014).

7. M. Satake, M. Enjoh, Y. Nakamura, et al., Biosci. Biotechnol. Biochem. 66 (2), 378-384 (2002).

8. M. Heyman and J. F. Desjeux, J Pediatr Gastroenterol Nutr 15 (1), 48-57 (1992).

9. L. Barthe, J. Woodley and G. Houin, Fundam Clin Pharmacol 13 (2), 154-168 (1999).

10. H. Teschemacher, Curr. Pharm. Des. 9 (16), 1331-1344 (2003).

11. W. Zhang, J. Miao, S. Wang and Y. Zhang, PLoS One 8 (5) (2013).

12. C. N. S. McLachlan, Medical Hypotheses 56 (2), 262-272 (2001).

13. Y. Jinsmaa and M. Yoshikawa, Peptides 1999, 957-962 (1999).

14. R. Rojas-Ronquillo, A. Cruz-Guerrero, A. Flores-Nájera, et al., Int. Dairy J. 26, 147-154 (2012).

15. D. Regazzo, D. Mollé, G. Gabai, et al., Mol Nutr Food Res 54 (10), 1428-1435 (2010). 
16. E. E. Sterchi, J. R. Green and M. J. Lentze, Biochem Soc Trans 9, 130-131 (1981).

17. G. Picariello, P. Ferranti and F. Addeo, Food Res. Int. 88, 327-335 (2016).

18. P. Plaisancié, R. Boutrou, M. Estienne, et al., J. Dairy Res. 82 (1), 36-46 (2015).

19. P. Plaisancié, J. Claustre, M. Estienne, et al., J. Nutr. Biochem. 24, 213-221 (2013).

20. J. P. Ulmschneider, J. C. Smith, S. H. White and M. B. Ulmschneider, J. Am. Chem. Soc. 133, 15487-15495 (2011).

21. M. B. Ulmschneider, J. C. Smith and J. P. Ulmschneider, Biophys J. 98, L60L62 (2010).

22. E. T. Kaiser and F. J. Kézdy, Proc.Natl.Acad.Sci.USA 80, 1137-1143 (1983).

23. W. C. Wimley and S. H. White, Nat. Struct. Mol. Biol. 3, 842-848 (1996).

24. G. Mandalari, A. M. Mackie, N. M. Rigby, M. J. S. Wickham and E. N. C. Mills, Mol. Nutr. Food Res. 53, S131 -S139 (2009).

25. F. J. Moreno, A. R. Mackie and E. N. C. Mills, J. Agric. Food Chem 53, 98109816 (2005).

26. L. Schrödinger, (2.0).

27. S. Li and M. Hong, J. Am. Chem. Soc. 133 (5), 1534-1544 (2011).

28. W. Humphrey, W. Dalke and K. Schulten, J. Mol. Graphics 14 (1), 33-38 (1996).

29. M. J. Abraham, T. Murtola, R. Schulz, et al., Softwarex 1, 19-25 (2015).

30. G. Bussi, D. Donadio and M. Parrinello, J. Chem. Phys. 126, 014101 (014101014107) (2007).

31. H. J. C. Berendsen, J. P. M. Postma, W. F. van Gunsteren, A. DiNola and J. R. Haak, J.Chem.Phys. 81 (8), 3684-3690 (1984).

32. B. Hess, H. Bekker, H. J. C. Berendsen and J. G. E. M. Fraaije, J. Comput. Biol. 18, 1463-1472 (1997).

33. M. Parrinello and A. Rahman, J. Appl. Phys. 52 (12), 7182-7190 (1981).

34. M. B. Ulmschneider, J. P. F. Doux, J. A. Killian, J. C. Smith and J. P. Ulmschneider, J. Am. Chem. Soc. 132, 3452-3460 (2010).

35. S. H. White and W. C. Wimley, Annu.Rev.Biophys.Biomol.Struc. 28, 319-365 (1999).

36. C. H. Chen, G. Wiedman, K. A. and M. B. Ulmschneider, BBA-Biomembranes 1838, 2243-2249 (2014).

37. R. B. Best, X. Zhu, J. Shim, et al., J. Chem. Theory Comput. 8, 3257-3273, (2012).

38. A. D. MacKerell, Jr., D. Bashford, M. Bellott, et al., J.Phys.Chem.B 102 (18), 3586-3616 (1998).

39. A. D. MacKerell, Jr., M. Feig and C. L. Brooks, II, J.Comput.Chem. 25, 14001415 (2004).

40. A. D. MacKerell, M. Feig and C. L. Brooks, J. Am. Chem. Soc. 126 (3), 698-699 (2004).

41. J. P. Ulmschneider and M. B. Ulmschneider, J. Chem. Theory Comput. 5, 1803-1813 (2009).

42. W. L. Jorgensen, J. Chandrasekhar, J. D. Madura, R. W. Impey and M. L. Klein, J. Chem. Phys. 79 (2), 926-935 (1983).

43. A. Altis, M. Otten, P. H. Nguyen, R. Hegger and G. Stock, J. Chem. Phys. 128 (24), 06B620 (2008). 
44. E. Lindahl, M. J. Abraham, B. Hess and D. van der Spoel, (zenodo, 2020).

45. B. Hess, Phys. Rev. E 65, 031910-031910 (2001).

46. T. Mendes Ferreira, F. Coreta-Gomes, O. H. S. Ollila, M. J. Moreno, W. L. C. Vaz and T. Topgaard, Phys. Chem. Chem. Phys. 15, 1976--1989 (2013).

47. M. Andersson, J. P. Ulmschneider, M. B. Ulmschneider and S. H. White, Biophys J. 104, L12-L14 (2013).

48. Y. Wang, C. H. Chen, D. Hu, M. B. Ulmschneider and J. P. Ulmschneider, Nat. Commun. (7), 13535 (2016).

49. X. Zhao, F. Pan, H. Xu, et al., Chem. Soc. Rev. 39 (9), 3480 (2010).

50. A. Q. Zhou, C. S. O'Hern and L. Regan, Proteins 82 (10), 2574-2584 (2014).

51. A. Altis, P. H. Nguyen, R. Hegger and G. Stock, J. Chem. Phys. 126 (24), 244111 (2007).

52. W. Kabsch and C. Sander, Biopolymers 22, 2577-2637 (1983).

53. N. G. Zhdanova, E. A. Shirshin, E. G. Maksimov, I. M. Panchishin, A. M. Saletsky and V. V. Fadeev, Photochem Photobiol Sci 14 (5), 897-908 (2015).

54. N. G. Zhdanova, E. A. Shirshin, E. G. Maksimov, I. M. Panchishin, A. M. Saletsky and V. V. Fadeev, Photochem. Photobiol. Sci. 14 (5), 897-908 (2015).

55. J. C. Gumbart, M. B. Ulmschneider, A. Hazel, S. H. White and J. P. Ulmschneider, J. Membrane Biol. 251, 345-356 (2018).

56. J. M. Dyer and A. Grosvenor, in Food Structures, Digestion and Health, edited by M. Boland, M. Golding and H. Singh (Academic Press, San Diego, 2014), pp. 303-317. 


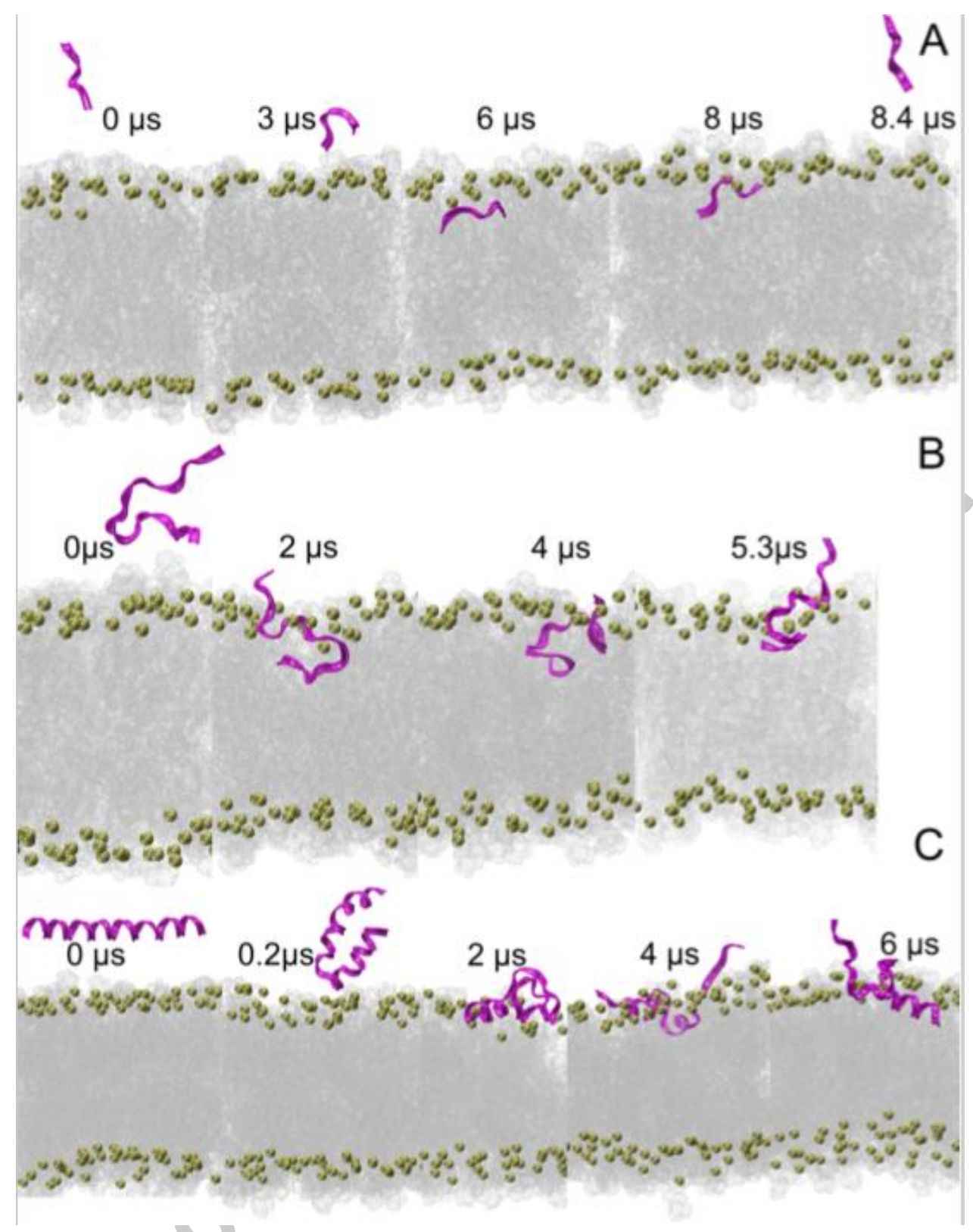

Figure 1. Snapshots of $\beta$-casein bioactive peptides. A. Insertion dynamics of the $\beta$-neocasomoprhin-6 (BCN6) at the POPC bilayer interface. The peptide enters and exit from the bilayer continuously during the $8.4 \mu$ s trajectory. B. The BCN17 attached to the POPC bilayer interface in few nanoseconds of trajectory. The peptide showed differential affinity for the lipid environment as the $\mathrm{N}$-terminus was inserted in the hydrophobic core, while the C-terminus was highly dynamics reentering to the bilayer several times during the simulation trajectory. C. BCN30, which is initially folded, lost its helical conformation within the first $\mu \mathrm{s}$. Continuous folding and unfolding was observed for this peptide, together with strong interactions of the C-terminus with the hydrophobic core. 

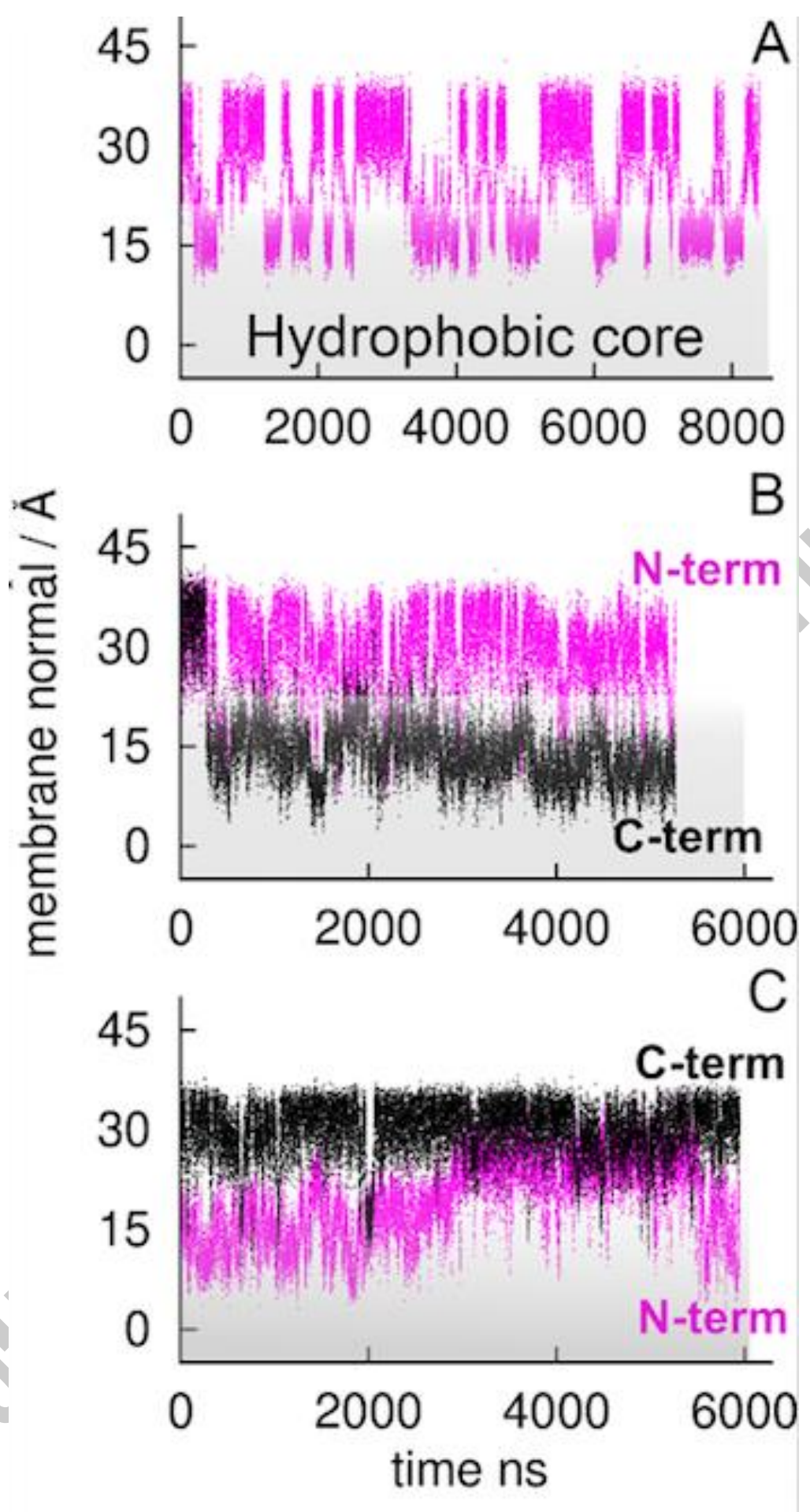

Figure 2. Position of the $\mathrm{C}$ - and $\mathrm{N}$-terminus of the $\beta$-casein bioactive peptides with respect to the membrane center $(z=0)$. A. Distance of the center of mass of BCN6 and the bilayer center. B. Distance of CBN17 C- and $\mathrm{N}$-terminus to the membrane center. $\mathrm{C}$. Distance of the CBN30 C- and $\mathrm{N}$ terminus to the bilayer center. The C- and N-terminus of BCN17 and BCN30 showed opposite affinity to the lipid bilayer. 


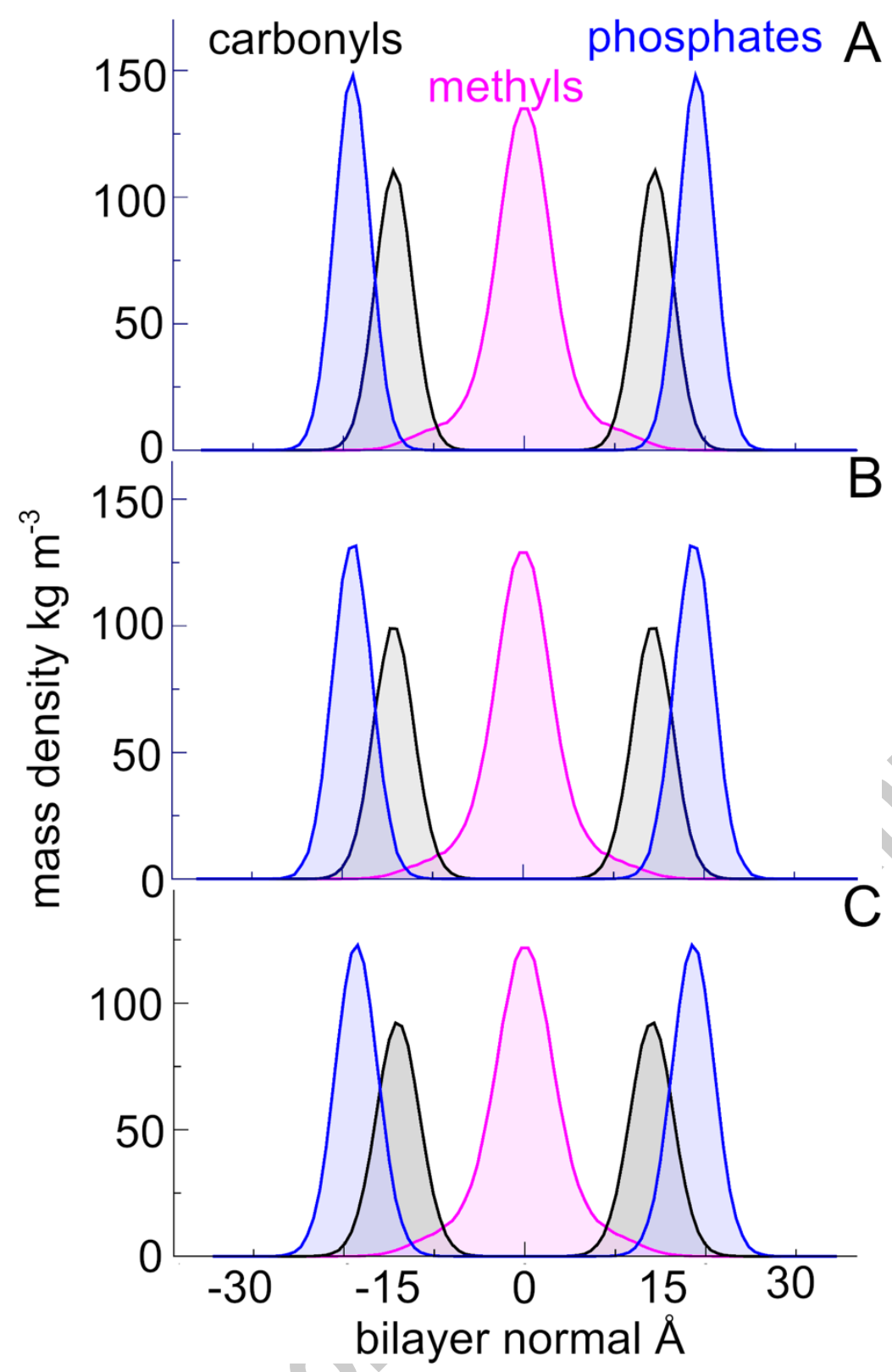

Figure 3. Mass density distribution for POPC lipid molecules across the bilayer. Color code: phosphate distribution-blue, carbonyl-black and terminal methyl groups-magenta. A. Hydrophobic core in the BCN6 simulation box highlighted by methyl groups. The bilayer Interfaces are highlighted by the phosphate and carbonyl distributions. B Hydrophobic core and interfaces in the BCN17 in simulation box. C Hydrophobic core and interfaces in the BCN30 in simulation box. 

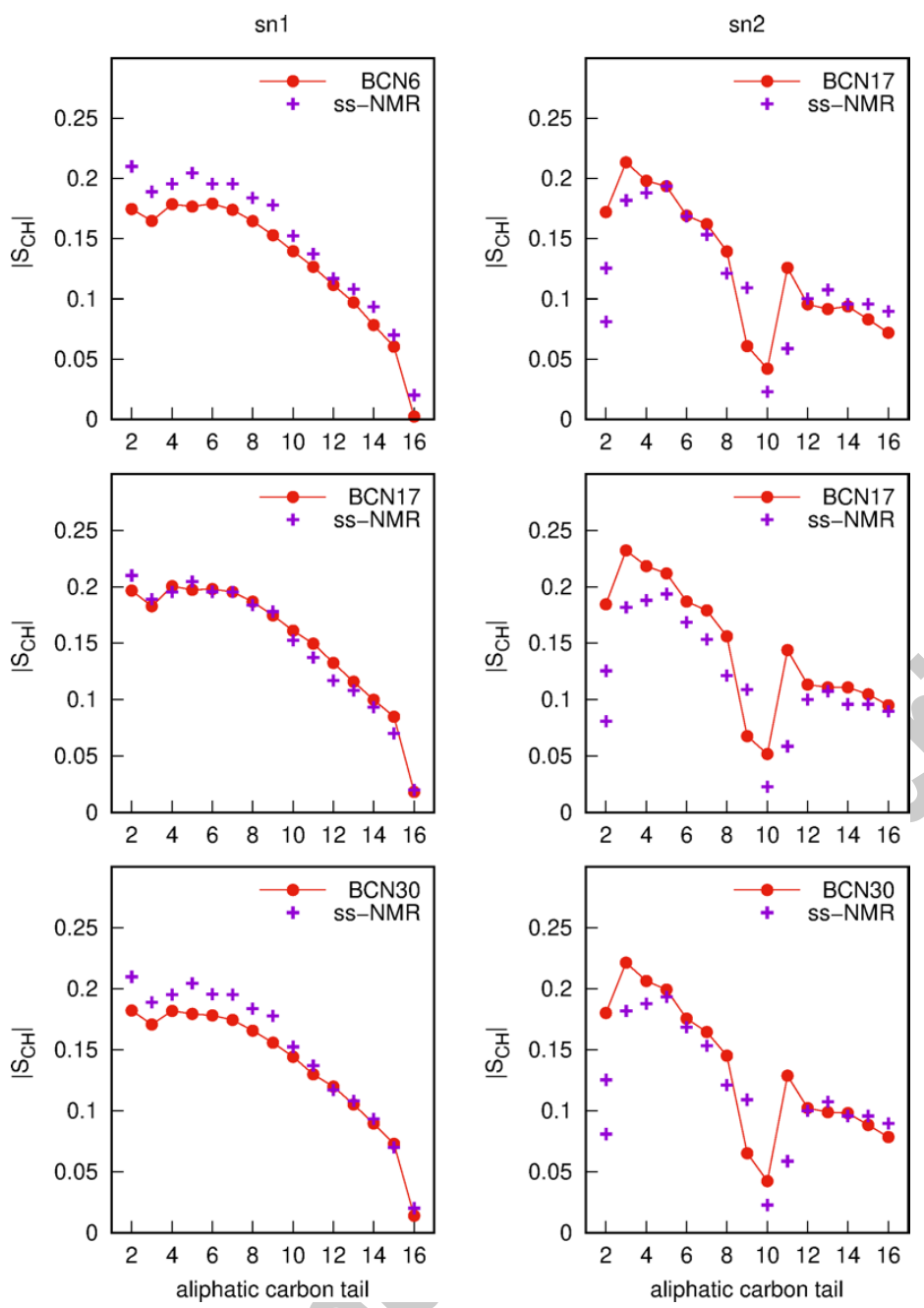

Figure 4. Absolute value of $\mathrm{S}_{\mathrm{CH}}$ order parameter for POPC lipid tails. Comparison of simulation results against experimental data obtained by solid state-NMR [46]. The lipid structure was well at $333 \mathrm{~K}$ in all the BCN systems. 


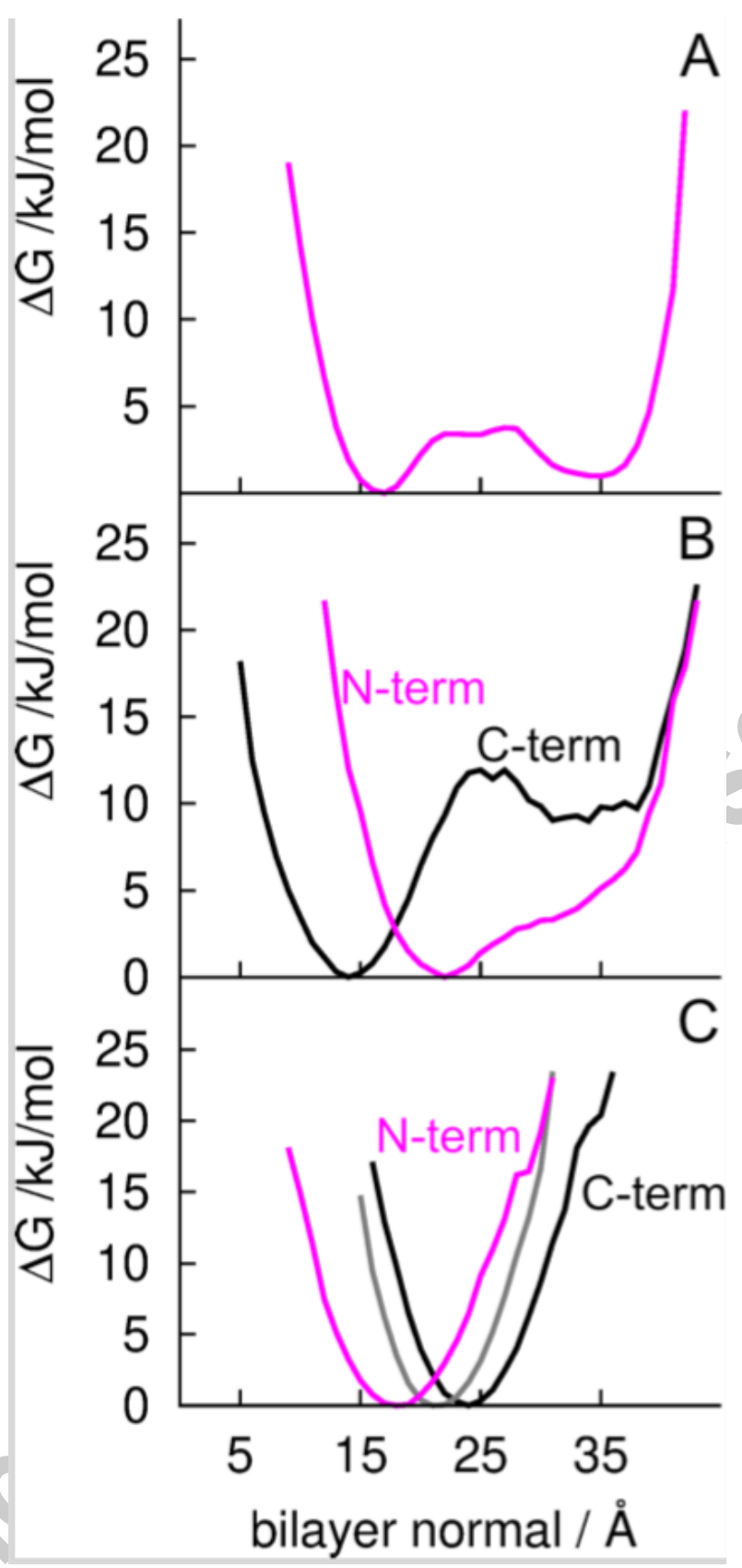

Figure 5. Internalization free energy profile: A. BCN6; B BCN 17; and C. BCN30 peptides. Relatively low barriers facilitate the membrane partitioning of short peptides. 

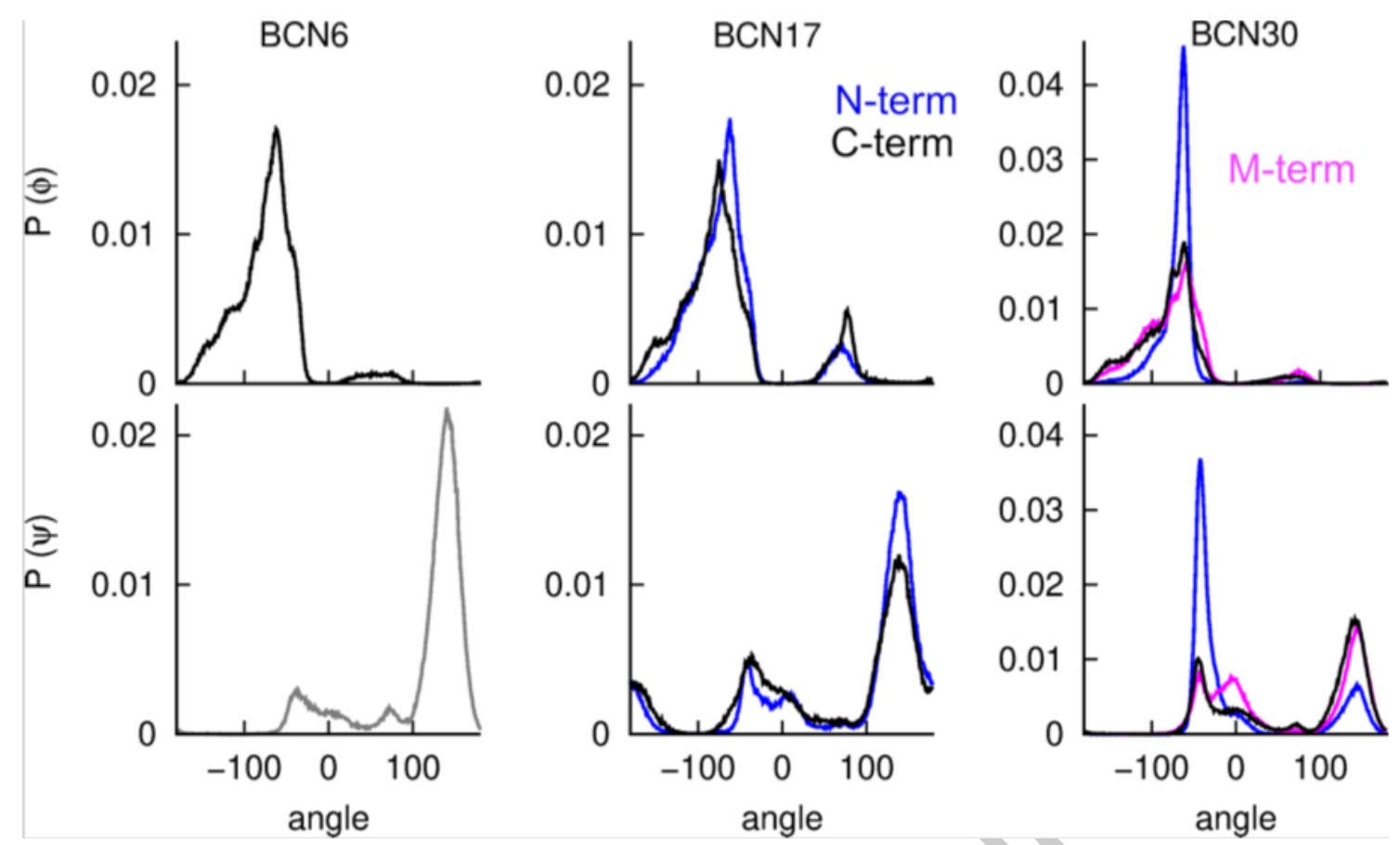

Figure 6. Distribution of the dihedral $\phi$ and $\psi$ angles of the backbone atoms of the $\mathrm{BCN}$ peptides. $\mathrm{N}$-term and $\mathrm{C}$-term fragments were defined for the CBN17 peptide, respectively, including Y193 to V201, and R202 to V 209. $\mathrm{N}$-term, M-term, and C-term fragments were defined for the BCN30 peptide, respectively, G94 to A103, P104 to K113, and Y114 to Q123. 

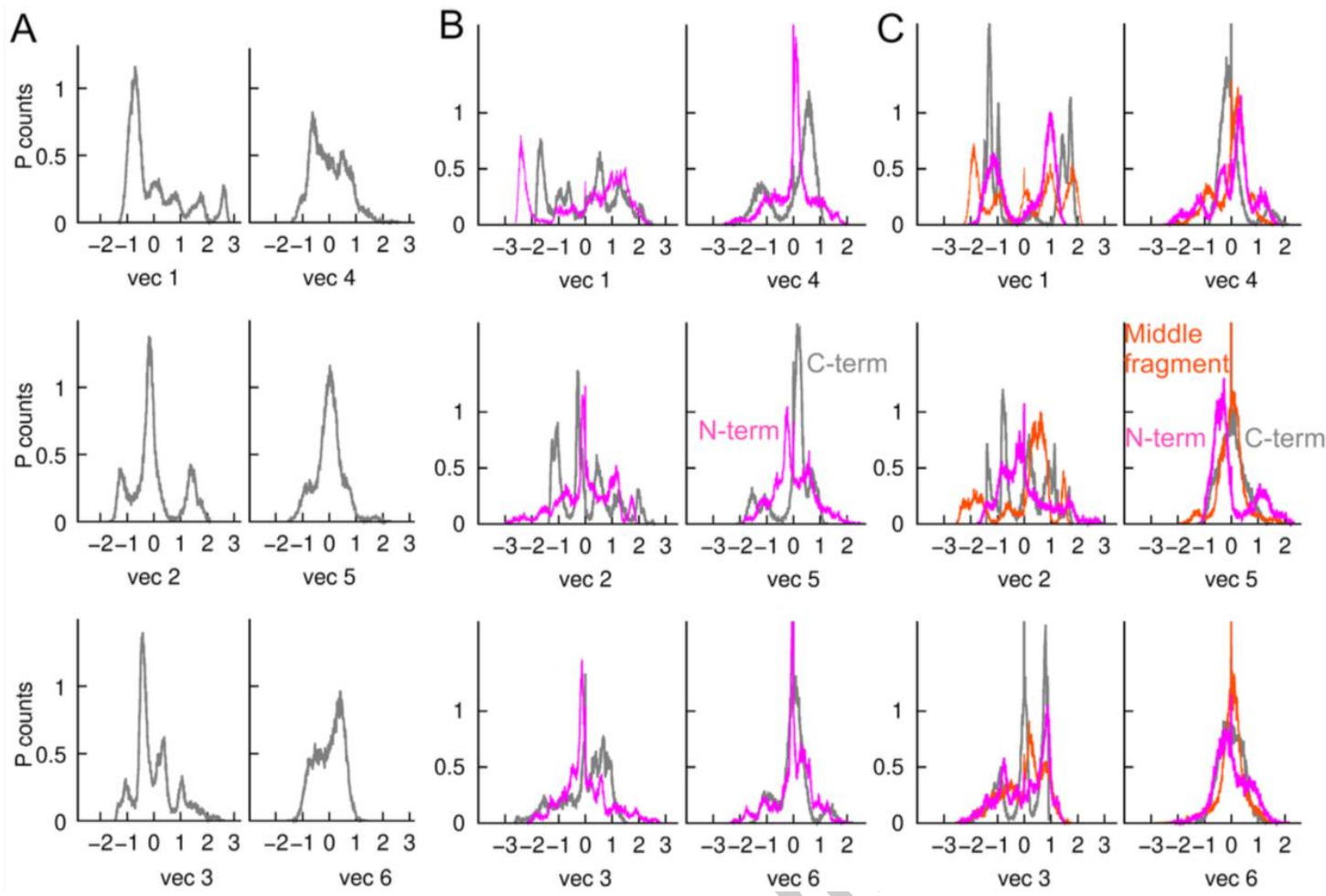

Figure 7. Distributions for the first six dihedral principal components of the BCN peptides. A. Dihedral principal components for $\phi$ and $\psi$ angles of backbone of BCN6, from Y114 to Y119. B. Dihedral principal components of BCN17 for $\phi$ and $\psi$ angles of the $\mathrm{N}$-terminus half (magenta solid line), from Y193 to V201, ant the C-terminus half (gray solid line), from R202 to V209. C. Dihedral principal components of $\mathrm{BCN} 30$ for $\phi$ and $\psi$ angles of the $\mathrm{N}$-terminus fragment (magenta solid line), from G94 to A103, the middle fragment (orange solid line), from P104 to K113, and the C-terminus fragment (gray solid line), from Y114 to Q123. Multimodal profiles were detected mainly in the first four principal components in all fragments. 


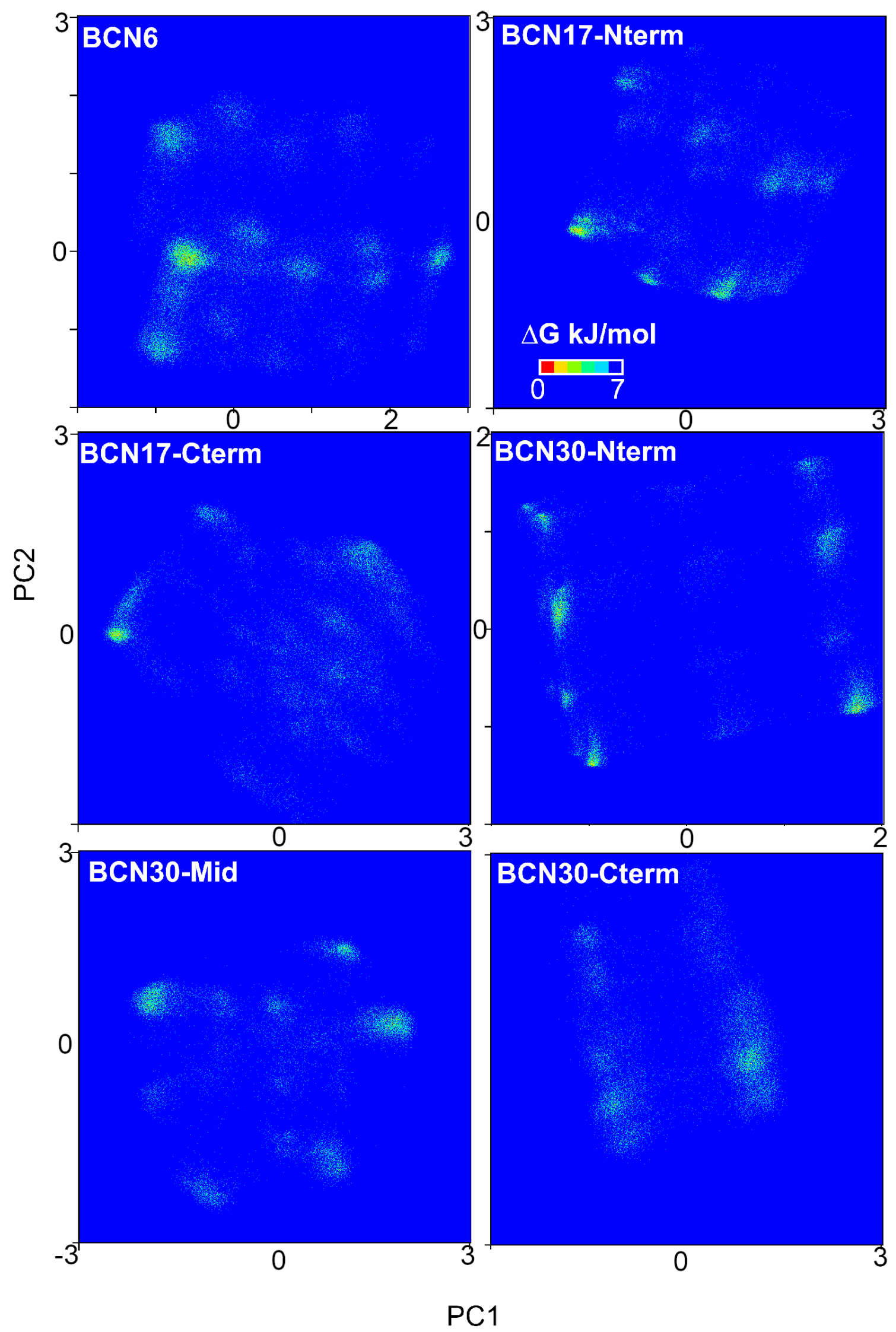

(C) 2020 Springer Science+Business Media, LLC, part of Springer Nature 
Figure 8. Conformational free energy landscape of the $B C N$ peptides.

Roughness of the conformational space described by dihedral backbone angles of the casein peptides; BCN6, BCN17 and BCN30. Each panel corresponds to a projection of the complete trajectories on principal components 1 (PC1) and 2 (PC2). In BNC17 the projection was calculated for two halves, the N-term and the C-term, and in BCN30 three fragments were analyzed, N-term, Mid-Term, and C-term.
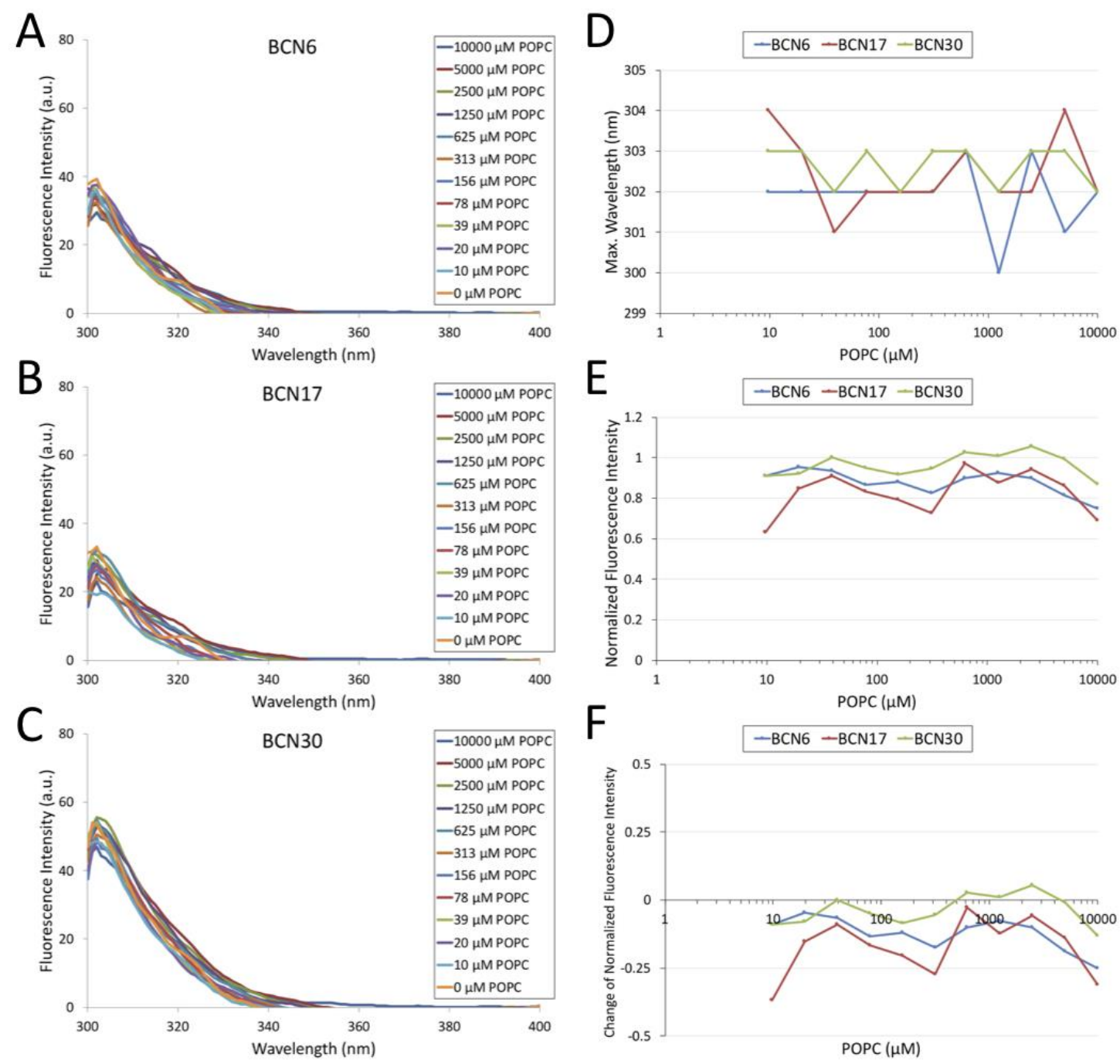

Figure 9 Tyrosine fluorescence probing of the peptide-lipid interactions. Fluorescence spectra of A. BCN6; B. BCN17; and C. BCN30. The peptide concentrations were $50 \mu \mathrm{M}$. D. Maximum wavelength of the fluorescence spectra at varied lipid (POPC large unilamellar vesicle) concentrations. E. Maximum fluorescence intensity of BCN6 (blue), BCN17 (red), and BCN30 (green) as a function of POPC concentration. The maximum fluorescence intensity was normalized to the value at which POPC concentration is 0 . F. Change of normalized fluorescence intensity. The change of normalized fluorescence intensity was subtracted by 1 (normalized fluorescence intensity at POPC concentration is 0 ). 